\title{
Adult T Acute Lymphoblastic Leukemia
}

National Cancer Institute

\section{Source}

National Cancer Institute. Adult T Acute Lymphoblastic Leukemia. NCI Thesaurus. Code C9142.

An acute T-lymphoblastic leukemia occurring in adults. 